\title{
Characteristics of Noises in High-Temperature YBCO Josephson Junctions
}

\author{
H.E. Horng, S.Y. Yang, and J.T. Jeng \\ Department of Physics, National Taiwan Normal University, Taipei 117, Taiwan \\ J.M. Wu, J.H. Chen, and H.C. Yang \\ Department of Physics, National Taiwan University, Taipei 106, Taiwan
}

\begin{abstract}
In this work, the characteristics of the noises for hightemperature YBCO Josephson junctions and SQUIDs were studied. The noise power spectrum densities showed complicated behaviors, which may be attributed to many causes. In order to realize the contributions of these causes to the noise, the noise power spectra were detected at various bias currents. Thus, the noises owing to the critical current fluctuation, resistance fluctuation or apparent flux motion can be investigated. By comparing these noise power spectra under the different conditions, the origins of the noises for the junctions and SQUIDs can be clarified.
\end{abstract}

\section{INTRODUCTION}

Because of the great potential applications of Josephson junctions and the superconducting quantum interference devices (SQUIDs), tremendous efforts have been put into investigating the characteristics of the high- $T_{c}$ Josephson junctions and SQUIDs [1-3]. Due to the high sensitivity, the junctions and SQUIDs were used as sensors, such as the biomagnetometers and the picovoltmeters [4,5], for probing tiny signals. Thus, the noises generated by the junctions and SQUIDs themselves may become comparable with the detected signals. Some experimental results showed that the low and white noise power spectra were found for both the junctions and SQUIDs at high frequencies, while a typical $1 / \mathrm{f}$-behavior can be observed at low frequencies. This $1 / \mathrm{f}$ noise confined the low-frequency application of the junctions or the SQUIDs. Many reported studies indicated that the $1 / f$ noise for the junctions and SQUIDs may arise from many causes $[6,7]$. To clarify the origins of the noise, the noise power spectrum densities were measured with various bias currents for the step-edge Josephson junction and the bicrystal YBCO SQUID in this work. By comparing the experimental results with the theoretical predictions, the contributions from different origins to the noises of the Josephson junctions and SQUIDs can be distinguished.

\section{EXPERIMENTAL DETAILS}

The $\mathrm{YBa}_{2} \mathrm{Cu}_{3} \mathrm{O}_{7-\mathrm{y}}$ single Josephson junction was obtained by patterning the YBCO film to be a $30 \mu \mathrm{m}$-wide microbridge on a step-edge $\mathrm{MgO}$ substrate. In order to probe the small variations in the voltage noise for the Josephson junction, an amplifier with a gain of 1000 was employed.

This work is supported by the National Science Council of ROC under grand Nos. NSC87-2112-M-003-008, NSC87-2112-M-003-001 and NSC87-2112$\mathrm{M}-002-002$
The voltage noise of the amplifier is around $10^{-16} \mathrm{~V}^{2} / \mathrm{Hz}$ at 1 Hz. Then, the amplified voltage noises of the junction were analyzed with a spectral analyzer (HP3562a). The SQUID investigated in this work is the washer-type $\mathrm{YBa}_{2} \mathrm{Cu}_{3} \mathrm{O}_{7-\mathrm{y}}$ bicrystal SQUID with an outer dimension of $90 \times 90 \mu \mathrm{m}^{2}$ and inner dimension of $20 \times 20 \mu \mathrm{m}^{2}$. Thus, the self-inductance of the SQUID is estimated to be $70 \mathrm{pH}$ [8]. For detecting the flux noises of the SQUID, the SQUID electronics (SQUID Catcher, NKT) with a flux-lock loop was used. Due to the usage of a flux-lock loop, a modulation coil was needed and integrated with the SQUID. This modulation coil showed a mutual inductance of $43 \mathrm{pH}$ between the coil and the SQUID. In the experiment, the samples were inserted into a $\mathrm{Bi}_{2} \mathrm{Sr}_{2} \mathrm{Ca}_{2} \mathrm{Cu}_{2} \mathrm{O}_{\mathrm{y}}$ superconducting or a mu-metal can to depress the influence from the ambient magnetic fields. Furthermore, all the experiments were conducted inside an electromagnetically shielded room.

\section{RESULTS AND DISCUSSION}

The V-I curve of the $\mathrm{YBa}_{2} \mathrm{Cu}_{3} \mathrm{O}_{7-\mathrm{y}}$ (YBCO) step-edge single Josephson junction at $4.2 \mathrm{~K}$ was shown in Fig. 1. It is clear that the V-I curve exhibits the RSJ behavior with a critical current $I_{c}$ of $0.133 \mathrm{~mA}$ and a normal resistance $R_{n}$ of $1.4 \Omega$ as the bias current I lower than $0.6 \mathrm{~mA}$. With increasing $\mathrm{I}$, a second kink was observed at I around $0.6 \mathrm{~mA}$. The twokink structure in the V-I curve for a step-edge Josephson junction is attributed to the fact that a step-edge junction may be composed of two Josephson junctions in series.

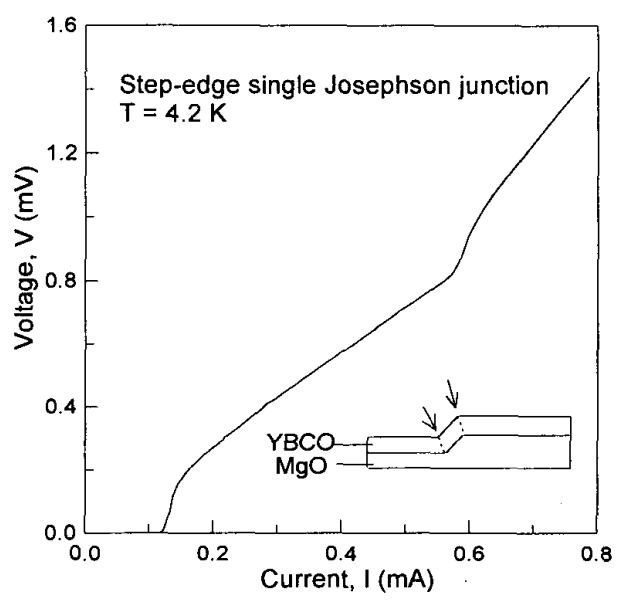

Fig, 1. V-I curve for the step-edge YBCO Josephson junction at $4.2 \mathrm{~K}$. The inset is the geometry of the step-edge junction. 


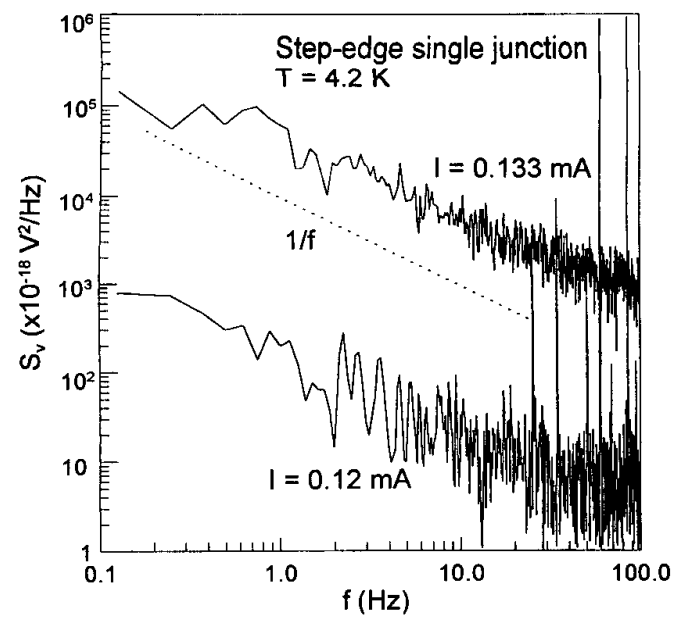

Fig. 2. The voltage noise power spectrum densities $S_{v}$ with various bias currents for the YBCO step-edge single Josephson junction.

The two grain boundaries are at the upper and lower corners of the step, as indicated in the inset of Fig. 1. Hence, at lower bias currents $(\mathrm{I}<0.6 \mathrm{~mA})$, the voltage is due to the junction with a smaller critical current. As the bias current approaching to 0.6 $\mathrm{mA}$, the second junction starts to become normal and the voltage at higher bias currents $(\mathrm{I}>0.6 \mathrm{~mA}$ ) results from both of the two junctions. By inspecting carefully the experimental result shown in Fig. 1, the normal resistance at bias currents higher than $0.6 \mathrm{~mA}$ was found to be $2.5 \Omega$. This reveals that the normal resistances for the two junctions in series are $1.4 \Omega$ and $1.1 \Omega$, respectively.

To investigate the characteristics of the noises for the stepedge Josephson junction, the voltage noise power spectrum densities $S_{v}(f)$ 's were measured for various bias current I's at $4.2 \mathrm{~K}$. The $\mathrm{S}_{\mathrm{v}}(\mathrm{f})$ curves show the background noise level of the amplifier at $\mathrm{I} \leq 0.12 \mathrm{~mA}$. The $\mathrm{S}_{\mathrm{v}}(\mathrm{f})$ curve with $\mathrm{I}=0.12 \mathrm{~mA}$ is shown in Fig. 2. Raising I close to $0.133 \mathrm{~mA}$, the whole $S_{v}$ curve moves abruptly to the region with larger values for $S_{v}$ and exhibits the highest noise level at $\mathrm{I}=0.133 \mathrm{~mA}$. The voltage noise spectrum with I being $0.133 \mathrm{~mA}$ displays a $1 / \mathrm{f}^{\alpha}$ dependence with $\alpha \sim 1$, as shown in Fig. 2. It was found that the value of $S_{\mathrm{v}}$ at $100 \mathrm{~Hz}$ is changed from $4 \times 10^{-18} \mathrm{~V}^{2} / \mathrm{Hz}$ to $4 \mathrm{x}$ $10^{-16} \mathrm{~V}^{2} / \mathrm{Hz}$. By increasing the bias current $I$, the $S_{v}$ curve is shifted downward and then almost remains unchanged as $I$ is increased up to $0.55 \mathrm{~mA}$. When I approaches to $0.6 \mathrm{~mA}$, the voltage noise starts to become larger and shows a local maximum at I around $0.6 \mathrm{~mA}$. To illustrate clearly the current dependence of the $S_{v}$ for the step-edge Josephson junction, the $\mathrm{S}_{\mathrm{v}}$ at $100 \mathrm{~Hz}$ for various bias currents are shown in Fig. 3(a). It is obvious that two peaks appear at $\mathrm{I}=0.133 \mathrm{~mA}$ and $0.6 \mathrm{~mA}$ which correspond to the critical currents of the two junctions in serious for the step-edge Josephson junction shown in Fig. 1. This coincidence implies that the variations in the voltage noise with the bias current may arise from the critical current fluctuation for the step-edge Josephson junction. To clarify this point, a further analysis was performed.

It is well known that the voltage noise for a Josephson junction can be due to the fluctuation of the critical current

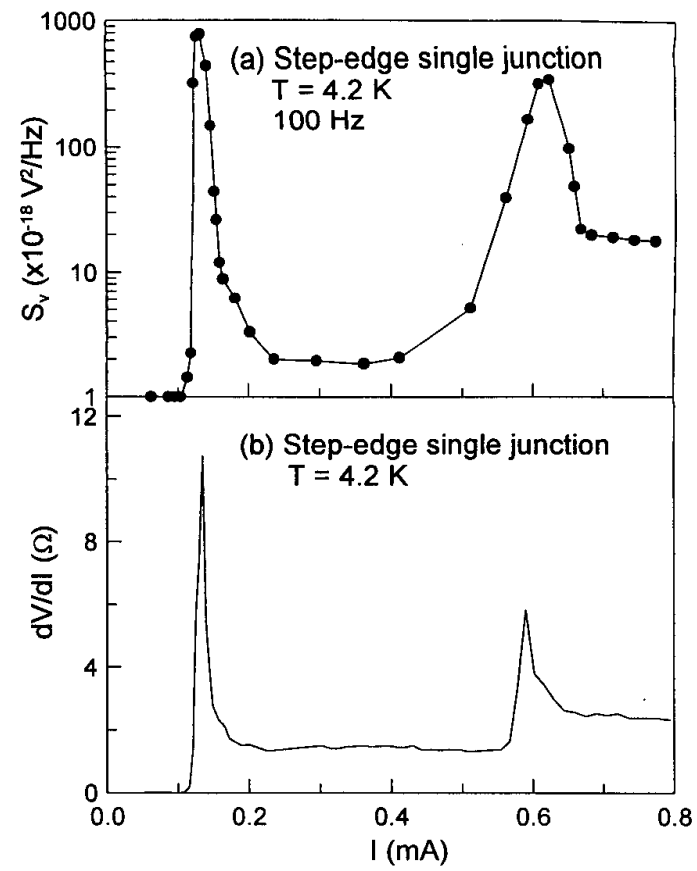

Fig. 3. (a) the bias current dependent $S_{v}$ at $f=100 \mathrm{~Hz}$ for the step-edge single junction. (b) the dynamic resistance as a function of the bias current at $4.2 \mathrm{~K}$ for the single junction.

and the normal resistance. Within a small signal analysis based on the RSJ model, the voltage noise $\mathrm{S}_{\mathrm{v}}$ can be quantitatively described. By neglecting for the moment correlation between the critical current fluctuation $\delta \mathrm{I}_{c}$ and the normal resistance fluctuation $\delta \mathrm{R}_{\mathrm{n}}$, we get

$$
S_{v}(f, I)=\left(V-R_{d} I\right)^{2} S_{I}(f)+V^{2} S_{R}(f),
$$

where $R_{d}=d V / d I$ is the dynamic resistance, $S_{I}=\left|\delta I_{c} / I_{c}\right|^{2}$ and $S_{R}$ $=\left|\delta R_{n} / R_{n}\right|^{2}$. The first term on the right-hand side in equation (1) is caused by the critical current fluctuation and the second term is generated from the normal resistance fluctuation. As shown in equation (1), the voltage resulted from the normal resistance fluctuation varies with the voltage quadratically. This disagrees with our experimental results shown in Fig. 3(a). On the other hand, $S_{v}$ due to the critical current fluctuation is deeply related to the dynamic resistance $R_{d}$ and also governed by the $R_{d}$ nonohmic dissipation state because of the large of $\mathrm{dV} / \mathrm{dI}$. To examine the relationship between the two bias current dependences of the $R_{d}$ and $S_{v}(f=100 \mathrm{~Hz})$ for the step-edge YBCO Josephson junction, the experimental $R_{d}$-I curve, which was obtained from the V-I data and shown in Fig. 3(b), was compared with the $\mathrm{S}_{\mathrm{v}}-\mathrm{I}$ curve. As shown in Figs. 3(a) and (b), the currents corresponding to local maximum noise levels happen to coincide with those at which two peaks occur in the $R_{d}$-I curve. In addition, a highly similar bias current modulation can be found for these two curves. This suggests that the critical current fluctuation dominate the variations in the voltage noises with the bias current for the step-edge YBCO Josephson junction at $4.2 \mathrm{~K}$.

The magnetic field modulated voltages $(\mathrm{V}-\Phi)$ for the 


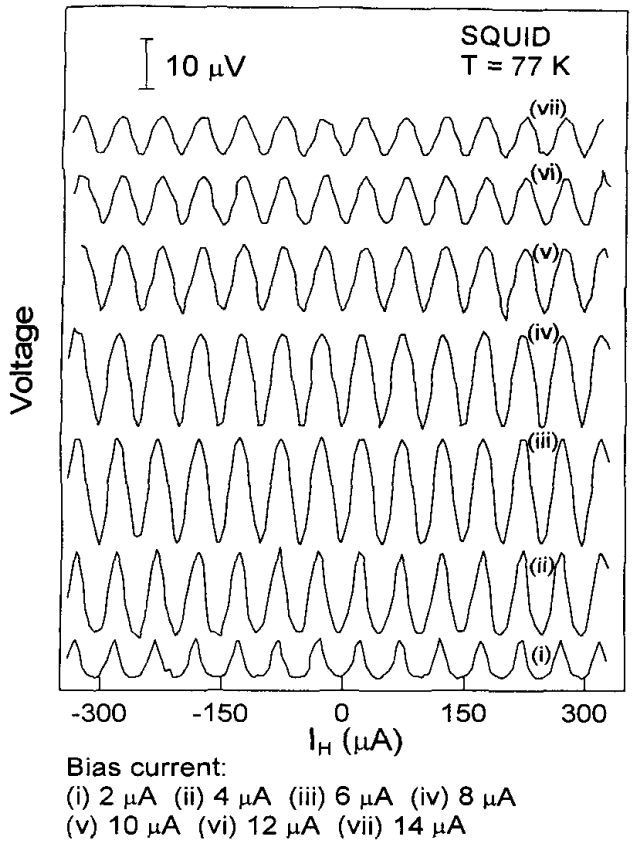

Fig. 4. The magnetic field modulated voltage oscillation of the bicrystal YBCO SQUID at $77 \mathrm{~K}$ under various dc bias currents.

bicrystal YBCO SQUID with $I_{c}=3 \mu \mathrm{A}$ and $R_{n}=8.7 \Omega$ were detected with various bias currents at $77 \mathrm{~K}$ shown in Fig. 4 . The maximum peak-to-peak voltage $\mathrm{V}_{\mathrm{pp}}$ occurs at bias current $\mathrm{I}$ around $6 \mu \mathrm{A}$. The flux noise power density spectra $S_{\Phi}(\mathrm{f})$ of the SQUID were measured at $77 \mathrm{~K}$ under various $\mathrm{dc}$ bias currents from $1.5 \mu \mathrm{A}$ to $14 \mu \mathrm{A}$ and were presented in Fig. 5 . For lower frequencies ( $\mathrm{f}<100 \mathrm{~Hz}$ ), the spectra exhibit approximately the $1 / \mathrm{f}$ behavior for $\mathrm{S}_{\Phi}$, whereas white noise spectra were observed at higher frequencies ( $f>100 \mathrm{~Hz}$ ). In addition, as the bias current is increased, the full spectrum moves toward a minimum flux noise level with $\mathrm{S}_{\Phi}(\mathrm{f}=1000 \mathrm{~Hz})$ $=2.25 \times 10^{-10} \Phi_{0}{ }^{2} / \mathrm{Hz}$ at $\mathrm{I}=6 \mu \mathrm{A}$. And then, the spectrum turns to display a higher flux noise level for $\mathrm{I}>6 \mu \mathrm{A}$. The $\mathrm{S}_{\Phi}$ 's at 6 $\mathrm{Hz}$ and $1000 \mathrm{~Hz}$ at various bias currents are shown in Fig. 6(a). For comparison, the $\mathrm{V}_{\mathrm{pp}}$ as a function of the bias current $\mathrm{I}$ is also shown in 6(b). It is worthy to note that optimum operation for the SQUID corresponds to the maximum $\mathrm{V}_{\mathrm{pp}}$ and minimum $\mathrm{S}_{\Phi}$.

To identify the origins of the measured $1 / \mathrm{f}$ flux noise through a flux-lock scheme for the SQUID, the experimental results were compared with the theoretical predictions. According to the reported studies, there are at least three possible sources for the $1 / \mathrm{f}$ flux noise spectrum $\mathrm{S}_{\Phi}$. First, the so-called "apparent flux noise" arising from the thermal motion of the vortices in the body of the SQUID [6]. Secondly, the out-of-phase critical current fluctuation in the two junctions of the SQUID [7]. Thirdly, the resistance fluctuation in the two junctions [7]. Koch et al. indicated that the flux noise caused by the thermally activated vortex motion should increase as the bias current is increased [9]. This is not consistent with the measured current dependent $1 / \mathrm{f}$ flux noise. Thus, the "apparent flux noise" is ruled out.

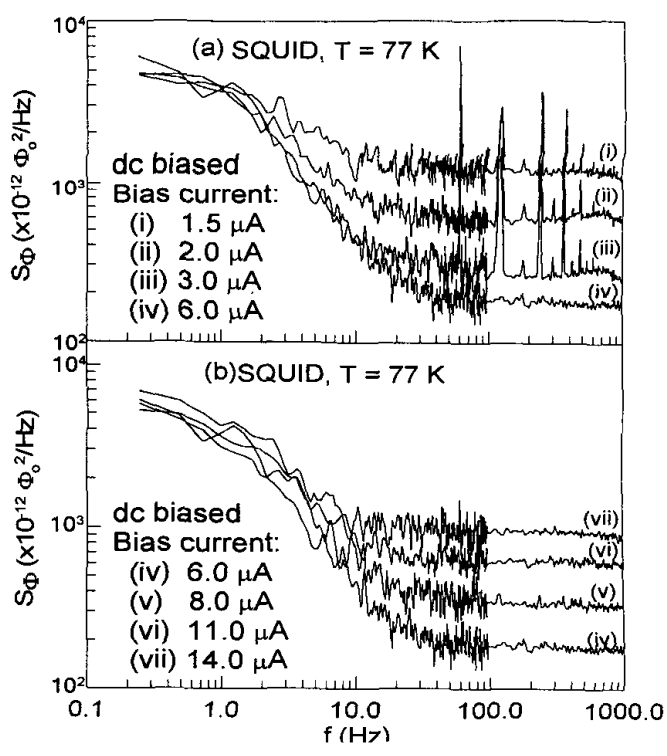

Fig. 5. Flux noise density spectra of the SQUID at $77 \mathrm{~K}$ for various dc biased currents.

An intuitive way to understand the effect due to the critical current fluctuation is to image that an out-of-phase critical current fluctuation $\Delta \mathrm{I}_{\mathrm{c}}$ occurs for the two junctions of the SQUID. This fluctuation will generate a circulating current for the SQUID and then cause the flux fluctuation via the relation $\Delta \Phi=\mathrm{L}_{\Delta \mathrm{l}}$, where $\mathrm{L}$ is the SQUID self-inductance. Furthermore, the voltage noise $S_{v}$ for the SQUID can be obtained by using $S_{v}=S_{\Phi} V_{\Phi}^{2}$, where $V_{\Phi}(=d V / d \Phi)$ is the transfer function. Since the $S_{\Phi}$ resulted from the out-of-phase critical fluctuation is approximately independent of the bias current, hence the $S_{v}$ 's with the same $V_{\Phi}$ but various bias currents should exhibit the same value. The experimental $S_{v}$ at $6 \mathrm{~Hz}$ versus the transfer function $V_{\Phi}$ is shown in Fig. 7 in

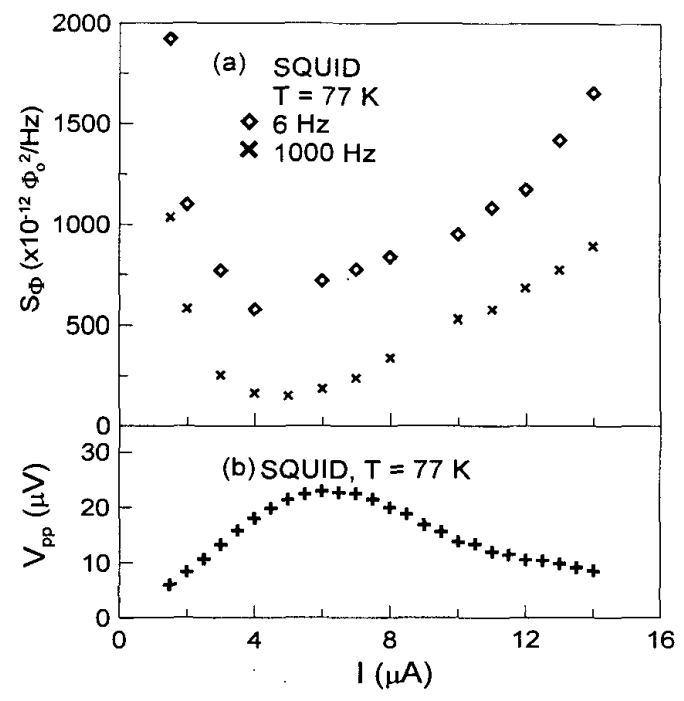

Fig. 6. $S_{\phi}$ and peak-to-peak voltage $V_{p p}$ versus the bias current $I$ at $77 \mathrm{~K}$ for the SQUID in (a) and (b), respectively. 


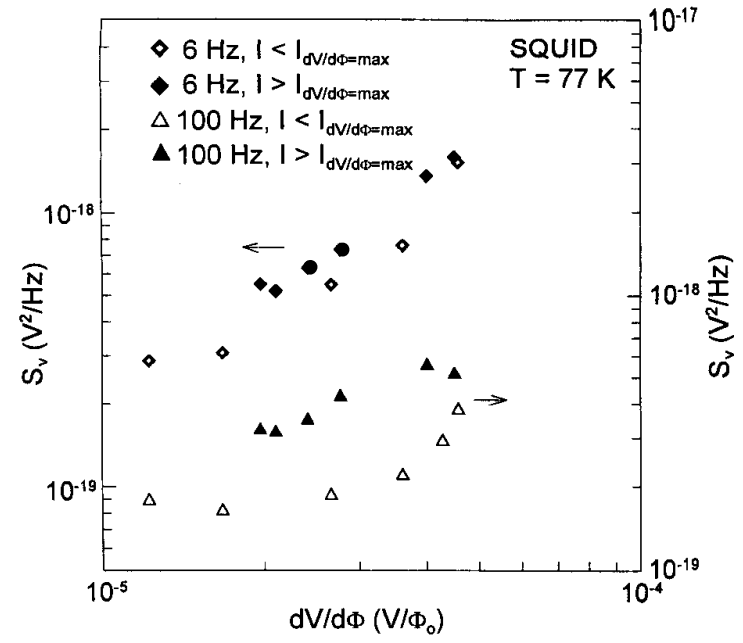

Fig. 7. The voltage noise $S_{\mathrm{v}}$ versus the transfer function $\mathrm{dV} / \mathrm{d} \Phi$ for the bicrystal YBCO SQUID at $77 \mathrm{~K}$.

which the $V_{\Phi}$ is varied by changing the bias current. It was observed to find that the voltage noises with the same $V_{\Phi}$ but different bias currents almost coincide with each other. This implies that the $1 / \mathrm{f}$ flux noise of the SQUID at low frequencies is attributed mainly to the critical current fluctuation. According to Koch et al. [6], the noise caused by the critical current fluctuation for the SQUID can be reduced greatly by using the bias reversing technique. This was demonstrated experimentally and shown in Fig. 8. It was observed that noise is depressed and the 1/f flux noise of the SQUID diminishes greatly under the bias reversing scheme. In Fig. 8, the white noise is also reduced. The reason for the reduction is still unclear and more investigations are undergoing to clarify it.

In Fig. 7, the voltage noises $S_{v}$ for the bicrystal SQUID at $1000 \mathrm{~Hz}$ with various $V_{\Phi}$ 's were also shown. The $S_{v}$ 's with the same $V_{\Phi}$ were observed to depart from each other, and $S_{v}$ with a higher bias current displays a larger value. By concerning an asymmetric resistance fluctuation $\Delta \mathrm{R}$

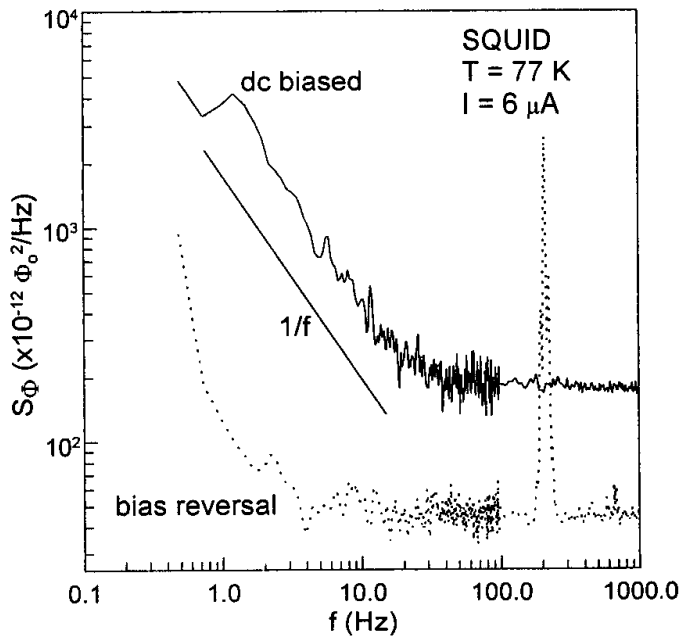

Fig. 8. The flux noises $S_{\Phi}$ 's with dc bias current and bias reversal. for the junctions of the SQUID as a source for the voltage fluctuation via the relation $\Delta \mathrm{V}=\mathrm{I} \Delta \mathrm{R}$, the voltage noise from the resistance fluctuation varies with the bias current. Thus, the SQUID voltage noises $S_{v}$ 's with two various bias currents corresponding to the same transfer function $V_{\Phi}$ will separate and the higher voltage noise can be found for the SQUID with a higher bias current. This result agrees with the experimental $\mathrm{S}_{\mathrm{v}}$ at $1000 \mathrm{~Hz}$ for the SQUID. As a result, the resistance fluctuation dominates the noises of the SQUID at higher frequencies.

\section{ConCLusion}

It is concluded that the variation in the $1 / \mathrm{f}$ voltage noise with the bias current is attributed mainly to the critical current fluctuation for the step-edge Josephson junction. The observed flux noises $\mathrm{S}_{\Phi}$ of the bicrystal YBCO SQUID can be classified as the low-frequency $1 / \mathrm{f}$ noise and the high-frequency white noise. By comparing the experimental results with the theoretical analysis based on different origins for the noises, the low-frequency noises are dominated by the out-of-phase critical current fluctuation in the junctions for the SQUID. Whereas the high-frequency noises of the SQUID mostly come from the resistance fluctuation in the junctions. In addition, the noises of the SQUID were reduced greatly by using the bias reversing technique.

\section{REFERENCES}

[1]E.J. Romans, T.G. Henrici, C. Carr, J.C. MacFarlane, C.M. Pegrum and G.B. Donaldson, "The properties of HTS Josephson junctions and DC SQUIDs fabricated on MgO bicystals", IEEE Trans. Appl. Supercond., 7, 2530-2533(1997)

[2]S.Y. Yang, H.E. Horng, W.L. Lee,H.W. Yu and H.C. Yang, "Fabrication of biepitaxial $\mathrm{YBa}_{2} \mathrm{Cu}_{3} \mathrm{O}_{7-y}$ Josephson junctions and SQUIDs", Chin. J. Phys., 36, 409(1998)

[3]J.Z. Sun, W.J. Gallagher, A.C. Callegari, V. Foglietti and R.H. Koch, "Improved process for high- $\mathrm{T}_{6}$ superconducting step-edge junctions", Appl. Phys. Lett., 63, 1561-1563(1993)

[4]P. Seidel, F. Schmidl, R. Weidl, S. Brabetz, F. Klemm, S. Wanderlich, L. Dörrer and $\mathrm{H}$. Mowak, "Development of a heart monitoring system based on thin film high- $T_{c}$ DC-SQUIDs", IEEE Trans. Appl. Supercond., 7, 3040-3043(1997)

[5]A.H. Miklich, D. Koelle, F. Ludwig, D.T. Nemeth, E. Dantsker and John Clarke, "Picovoltmeter based on high transition temperature SQUID", Appl. Phys. Lett., 66, 230-232(1995)

[6]R.H. Koch, J. Clarke, W.M. Goubau, J.M. Martinis, C.M. Pegrum and D.J. van Harlingen, "Flicker ( $1 / \mathrm{f})$ noise in tunnel junction dc SQUIDs", J. Low Temp. Phys., 51, 207-224(1983)

[7]J. Chen, T. Ogawa, H. Nakamura, H. Myoren, K. Nakajima and T.

Yamashita, "Low frequency $(1 / \mathrm{f})$ noise in $\mathrm{YBa}_{2} \mathrm{Cu}_{3} \mathrm{O}_{7} \delta$ grain boundary junction dc superconducting quantum interference devices", J. Appl. Phys., 76, 1895-1898(1994)

[8]M.B. Ketchen and J.R. Clem, "DC SQUID flux focuser", SQUID'85, Superconducting Quantum Interference Devices and Their Applications: Proceeding of the Third International Confrence on Superconducting Quantum Interference Devices (1985)

[9]R.H. Koch, W. Eidelloth, B.Oh. R.P. Robertazzi, S.A. Andrek and W.J. Gallagher, "Identifying the source of $1 / \mathrm{f}$ noise in SQUIDs made from high-temperature superconductors", Appl. Phys. Lett., 60, 507509(1992) 